Nachhaltigkeit und Lebensmittelproduktion

\section{Bewertung von Unternehmen im Lebensmittelsektor}

Die Welternährungsorganisation FAO hat nun erstmals eine umfassende Definition von Nachhaltigkeit für den Lebensmittelsektor vorgelegt. Auf dieser Basis wurde mit SMART eine Methode entwickelt, anhand derer Unternehmen aus dem Lebensmittelbereich ihre Nachhaltigkeitsleistungen darstellen können. Von Christian Schader, Julia Jawtusch und Matthias Stolze

D as Thema Nachhaltigkeit wird für Unternehmen aus dem Lebensmittelbereich zunehmend wichtiger, um beispielsweise durch eine höhere Energieeffizienz Kosten einzusparen oder um sich von Mitbewerbern am Markt zu unterscheiden (Haanaes et al. 2011). Es gibt zwar eine Vielzahl von Nachhaltigkeitsbewertungs- und Umweltmanagementsystemen, jedoch wird der Begriff Nachhaltigkeit inflationär und uneinheitlich gebraucht. Darüber hinaus besteht keine Möglichkeit, die Nachhaltigkeitsleistung von verschiedenen Unternehmen zu vergleichen.

\section{Internationale Leitlinie zur Nachhaltigkeit im Lebensmittelsektor}

Um dem entgegenzuwirken hat die Welternährungsorganisation FAO im Juni 2012 die sogenannten SAFA-Leitlinien veröffentlicht (FAO 2012). SAFA steht für „Sustainability Assessment of Food and Agriculture Systems“ oder Nachhaltigkeitsbewertung von Lebensmittelsystemen. Die SAFA-Leitlinien geben einen Rahmen vor, wie die Nachhaltigkeitsbewertung im Lebensmittelsektor durchzuführen ist. SAFA definiert vier Dimensionen der Nachhaltigkeit: Ökologie, Ökonomie, Soziales, Unternehmensführung. Die Dimensionen gliedern sich in 20 Themen und insgesamt etwa 60 Unterthemen. Damit existiert erstmals eine umfassende internationale Leitlinie zur Nachhaltigkeit, anhand derer vergleichende Analysen von Unternehmen des
Lebensmittelsektors durchgeführt werden können.

\section{Eine neue Bewertungs- methode}

Mit der Sustainability Monitoring and Assessment RouTine, kurz SMART, hat das Forschungsinstitut für biologischen Landbau (FiBL) auf der Basis der FAO-SAFA-Leitlinien eine Methode entwickelt, mit der die Nachhaltigkeit von Firmen im Lebensmittelsektor und von landwirtschaftlichen Betrieben bewertet werden kann. SMART besteht aus einer standardisierten Datenerfassungsroutine, einem Indikatorenset, Bewertungsprozeduren und einer Datenbank. Eine SMART-Bewertung erfolgt nach einer klar definierten Vorgehensweise in sechs Schritten: a) Festlegung der Ziele, b) Überprüfung der Kriterienrelevanz, c) kontextspezifische Festlegung des Indikatorensets, d) Datenerhebung, e) Datenanalyse und Bewertung und f) Erstellung des Bewertungsberichtes.

Daten, die bereits aus Umweltmanagementsystemen, der Biozertifizierung, Klimabilanzen oder Ökobilanzen vorliegen, lassen sich in das SMART-Indikatorensystem integrieren. Somit kann der Aufwand bei einer SMART-Analyse reduziert werden.

Die Ergebnisse einer SMART-Nachhaltigkeitsbewertung können Unternehmen zur Optimierung ihrer Betriebsabläufe, zur Bewertung der gesamten Wertschöpfungskette sowie für die Kommunikation gegenüber Kunden verwenden.
Landwirtschaftliche Betriebe werden mit dem SMART-Farm-Tool bewertet, das es erlaubt, innerhalb von ein bis drei Stunden (je nach Betriebsgröße und -struktur) die Nachhaltigkeitsleistungen zu bewerten. Somit können in kurzer Zeit eine große Anzahl von Betrieben bewertet werden. Daher eignet sich das Tool zum Lieferanten-Monitoring und zur Nachhaltigkeitszertifizierung.

\section{Schnelle Bewertung möglich}

SMART wurde in einer Pilotphase von März 2012 bis Oktober 2013 in fünf Lebensmittelunternehmen aus der Schweiz, Deutschland und Mexiko ausgiebig getestet. Um die breite Anwendbarkeit des Ansatzes sicherzustellen, wurden Verarbeitungs- und Handelsunternehmen unterschiedlicher Branchen betrachtet. SMART und die ersten Unternehmenserfahrungen wurden der Öffentlichkeit am 24. Oktober 2013 in Frankfurt vorgestellt.

\section{Literatur \\ FAO (2012): Guidelines for Sustainability Assess- ment in Food and Agriculture (SAFA). Rome, Food and Agriculture Organization of the United Nations (FAO). \\ Haanaes, K. et al. (2011): Sustainability: the ,embracers' seize advantage. Findings from the 2010 Sustainability \& Innovation Global Executive Study and Research Project. MIT Sloan Management Review and The Boston Consulting Group.}

AUTOREN + KONTAKT

Dr. Christian Schader ist Geschäftsführer der Sustainable Food Systems Society $\mathrm{GmbH}$ und zuständig für die Entwicklung und Vermarktung von SMART.

Dr. Christian Schader, Sustainable Food Systems Society $\mathrm{GmbH}$, Ackerstrasse 113, Postfach 219, 5070 Frick, Schweiz. Tel: +41628650416,

E-Mail: christian.schader@fibl.org Julia Jawtusch arbeitet am und Dr. Matthias Stolze leitet das Departement Sozioökonomie am Forschungsinstitut für biologischen Landbau (FiBL).

FiBL, Ackerstrasse 113, Postfach 219 5070 Frick, Schweiz. Tel: +41 628657255 , E-Mail: julia.jawtusch@fibl.org matthias.stolze@fibl.org 
Copyright (C) 2013, IÖW und oekom Verlag. Die Nutzung des Artikels ist Abonnenten von Ökologisches Wirtschaften vorbehalten. Nachdruck und Vervielfältigung des Artikels einschließlich Speicherung und Nutzung auf optischen und elektronischen Datenträgern nur mit Zustimmung der Redaktion von Ökologisches Wirtschaften (http://www.oekologischeswirtschaften.de). 